\title{
Stratégie et équipements de prévention vis-à-vis du gel de printemps et de la grêle. Perspectives en lien avec les changements climatiques, projet ADVICLIM
}

\section{Strategy and prevention equipment against spring frost and hail. Perspectives related to climate change, ADVICLIM project}

\author{
J. Rochard ${ }^{1}$, C. Monamy ${ }^{2}$, B. Pauthier ${ }^{3}$, et A. Rocque ${ }^{4}$ \\ ${ }^{1}$ IFV (Institut Français de la Vigne et du Vin), 17 rue Jean Chandon Moët, 51200 Epernay, France \\ 2 BIVB (Bureau Interprofessionnel des Vins de Bourgogne) 12 boulevard Bretonnière, 21200 Beaune, France \\ ${ }^{3}$ CIVC (Comité Interprofessionnel du Vin de Champagne) 5 rue Henri Martin, 51200 Epernay, France \\ ${ }^{4}$ Chambre d'Agriculture d'Indre-et-Loire, 38 rue Augustin Fresnel, 37171 Chambray les Tours Cedex, France
}

\begin{abstract}
Résumé. Une grande partie du vignoble a été touchée par l'épisode de gel qui est intervenu sur plusieurs jours fin avril 2017 et qui a occasionné des dégâts pour de nombreuses régions françaises y compris méridionales, ainsi que d'autres pays de la zone Ouest de l'Europe (Angleterre, Allemagne, Italie, Suisse, etc.). Le gel, la grêle et une sécheresse très marquée dans les zones méridionales ont abouti à une baisse d'environ 10 millions d'hectolitres par rapport à 2016 ce qui correspond, par rapport à une moyenne décennale, à une diminution de l'ordre de $18 \%$. En 2018, de nombreuses régions viticoles France ont été touchées par la grêle. Le projet CLIMFROST, financé par France Agrimer, a pour objectif d'aider la profession viticole française à appréhender les risques de gel de printemps et de grêle dans leur contexte local, afin d'adopter une stratégie (réserves climatiques, assurances, mesures agronomiques, techniques de protection) permettant de réduire l'impact financier direct et indirect. En complément des aspects techniques, la communication a pour objectif de resituer ces phénomènes climatiques extrêmes dans la perspective des changements climatiques, en lien notamment avec le projet européen LIFE ADVICLIM www.adviclim.eu. Cette communication présente une synthèse d'une plaquette «aléas climatiques de la vigne : grêle, gel de printemps », téléchargeable sur le site www. vignevin. com.
\end{abstract}

\begin{abstract}
A large part of the vineyard was affected by the freezing episode which occurred over several days at the end of April 2017 and which caused damage for many French and southern regions, as well as other countries in the western part of France. Europe (England, Germany, Italy, Switzerland, etc.). Frost, hail and a very pronounced drought in the southern areas resulted in a decrease of about 10 million hectoliters compared to 2016, which corresponds to a 10-year average decrease of about $18 \%$. In 2018, many French wine regions were hit by hail. The CLIMFROST project, funded by FranceAgrimer, aims to help the French wine industry to apprehend the risks of freezing spring and hail in their local context, in order to adopt a strategy (climate reserves, insurance, agronomic measures, farming techniques protection) to reduce the direct and indirect financial impact. In addition to the technical aspects, the communication aims to situate these extreme weather phenomena in the context of climate change, particularly in connection with the European project LIFE ADVICLIM. www .adviclim.eu. This paper presents a summary of a leaflet "climate risk of the vine: hail freezing spring" downloadable in French on the site www .vignevin.com.
\end{abstract}

\section{Introduction}

Une grande partie du vignoble a été touchée par l'épisode de gel qui est intervenu sur plusieurs jours fin avril 2017 et qui a occasionné des dégâts pour de nombreuses régions françaises y compris méridionales, ainsi que d'autres pays de la zone Ouest de l'Europe (Angleterre, Allemagne, Italie, Suisse, etc.). Le gel, la grêle et une sécheresse très marquée dans les zones méridionales ont abouti en France à une baisse d'environ 10 millions d'hectolitres par rapport à 2016, ce qui correspond par rapport à une moyenne décennale, à une diminution de l'ordre de $18 \%$ [1]. En 2018, un épisode orageux très violent a affecté plusieurs régions viticoles françaises et notamment le Bordelais et le Cognaçais (Figs. 1 et 2). C'est dans ce contexte qu'a été développé le projet CLIMFROST, coordonné par l'Institut Français de la Vigne et du Vin et financé par FranceAgrimer. Il a pour objectif d'aider la profession viticole française à appréhender les risques de gel de printemps et de grêle dans leur contexte local. Une plaquette est disponible sur le site www.vignevin.com. 


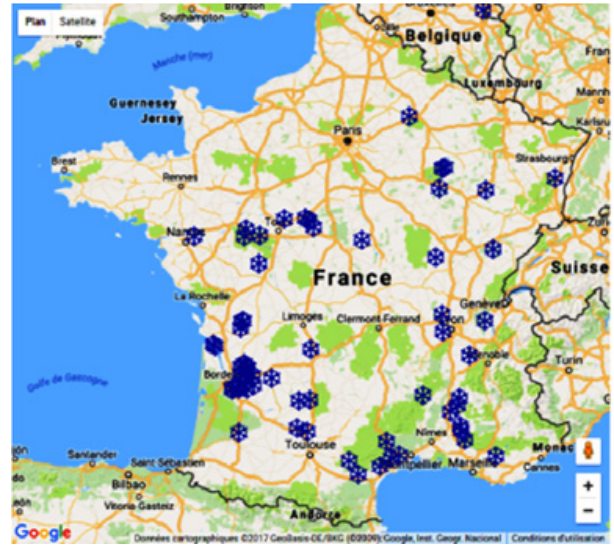

Figure 1. Cartes régions françaises touchées par le gel en 2017 (Source www.vitisphere.com).

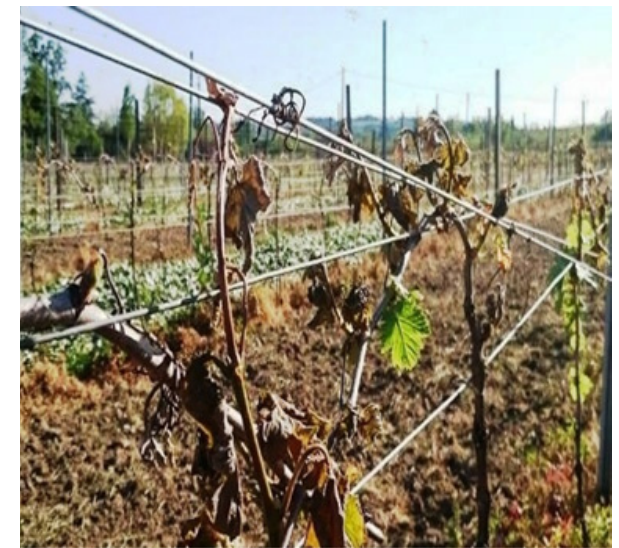

Figure 2. Dégâts de gel dans le bordelais en 2018 (Source www. avenir-aquitain.com).

Des mesures indirectes peuvent être envisagées pour réduire les aléas climatiques:

+ La souscription d'une assurance récolte, incluant le gel et la grêle. Le coût élevé de la cotisation constitue néanmoins un frein important au développement de ce type d'assurance. Par ailleurs le déficit de récolte peut conduire à un handicap commercial au cours des années suivantes, qui n'est généralement pas pris en compte par l'assurance.

+ La mise en place d'un dispositif de réserve 《climatique» alimenté au cours des bonnes années, permet de compenser le déficit des années déficitaires notamment en lien avec des accidents météorologiques (gel, grêle, sécheresse, etc.). La procédure de «réserve qualitative », initiée depuis longtemps en Champagne, se développe maintenant dans de nombreuses autres régions par le dispositif de Volume Complémentaire Individuel (VCI). C'est un volume de vin produit au-delà du rendement annuel de l'appellation et mis en réserve qui peut être utilisé ultérieurement, notamment à la suite d'aléas climatiques.

Parallèlement à ces mesures indirectes, la mise en place de dispositifs de protection peut être envisagée pour des régions avec une forte fréquence de risque.

\section{La grêle}

\subsection{Mécanisme}

Les dégâts sur vignes provoqués par des grains de glace d'au moins $5 \mathrm{~mm}$ de diamètre et d'une densité proche

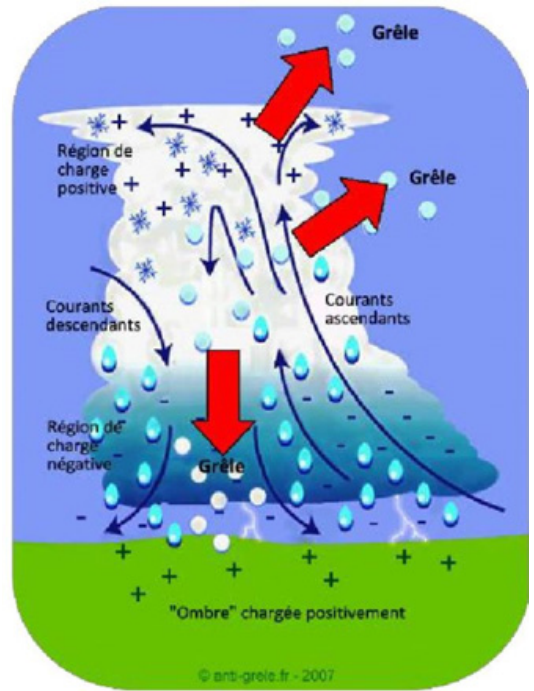

Figure 3. Schéma de la formation de la grêle (Source www. antigrele.fr).

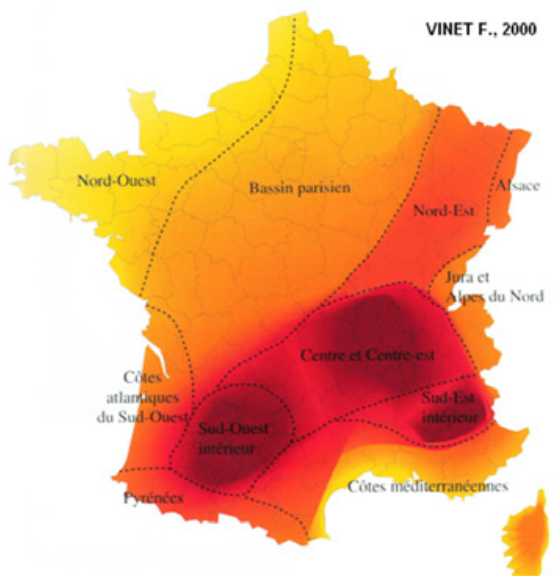

Figure 4. Fréquence de risque de grêle en France d'après F. VINET, Le risque grêle en agriculture éditions technique et documentation, 2000.

de 0.9 , selon la définition officielle, peuvent être très importants : défoliation, destruction des futurs sarments et des bourgeons. Elle peut affecter la récolte sur plusieurs années.

Avec de fortes différences de température entre l'air au-dessus du sol et le sommet de la troposphère (vers $12 \mathrm{~km}$ d'altitude), et lorsque les basses couches de l'atmosphère sont riches en humidité, des nuages de convection appelés cumulonimbus se développent pour transférer vers le haut le surplus d'énergie de la basse atmosphère. Au sein de ces nuages, des processus microphysiques vont provoquer la condensation de la vapeur d'eau en grêle. Avec peu de noyaux de congélation dans l'air, les cristaux de glace sont rares et les grêlons deviennent assez gros pour tomber à grande vitesse jusqu'au sol (Figs. 3 et 4 ).

Les « ingrédients »favorisant la formation d'orages sont souvent présents l'été. Il s'agit essentiellement d'air instable du sol jusqu'en altitude, d'humidité relativement élevée dans les bas niveaux, d'air sec en altitude, un facteur de soulèvement du courant d'air tels que les collines, montagnes ou encore l'approche d'un front froid. Les cumulonimbus, caractérisés par leur forme d'enclume, génèrent les plus gros grêlons. 
Comme le précise F. Vinet: «Pour qu'il y ait formation de grêlons, il faut une température inférieure à $0{ }^{\circ} \mathrm{C}$ (en réalité $-15^{\circ} \mathrm{C}$ ) dans le nuage. En France, en été, l'isotherme $0{ }^{\circ} \mathrm{C}$ se trouve en moyenne à 3 000, voire 4000 mètres d'altitude. Seuls les nuages à fort développement vertical sont capables de porter les gouttelettes d'eau à une telle altitude et de les y maintenir suffisamment longtemps pour que les grêlons aient le temps de se former $\gg$ [2].

En France, le couloir de fréquence d'orages, avec un potentiel de grêle, remonte majoritairement du Sud-ouest au Nord-est en passant par le Massif Central et la FrancheComté.

\subsection{Dispositifs de protection}

Face à des événements épisodiques, mis à part pour les filets, il est difficile de démontrer statistiquement l'efficacité des systèmes de limitation de l'impact de la grêle. Différents dispositifs, parfois développés notamment en arboriculture, canons acoustiques et fusées, sont assez peu développées en viticulture, en raison de des nuisances et/ou des contraintes réglementaires qui en résultent, ou encore parce que l'efficacité est remise en cause. Dans les régions viticoles ce sont majoritairement les dispositifs par ensemencement d'iodure d'argent qui sont le plus souvent privilégiés [3].

\section{- Protection par filet}

Le dispositif le plus efficace consiste à disposer le filet au-dessus des rangs, à plat, en «couverture totale». Ce système est adapté aux vignes larges (à partir de 3 mètres), car les filets doivent pouvoir s'ouvrir pour pouvoir déverser la grêle accumulée entre les rangs en cas de poids trop important. Ce système permet d'assurer les interventions manuelles et mécaniques dans la vigne mais il nécessite une infrastructure lourde pour l'installation, avec un impact esthétique. L'investissement est important, de l'ordre de 20 à $25000 €$ par hectare pour un écartement de rang de 3 mètres. Ce dispositif, non autorisé en France pour les vins sous indication géographique, est généralement réservé aux raisins de table.

Pour les vignes dont l'écartement est inférieur à 3 mètres, les filets monorangs ( «rang par rang») sont plus adaptés. Les filets anti-grêle comportent un maillage (mailles carrées de $5 \times 5 \mathrm{~mm}$ ou $7 \times 3 \mathrm{~mm}$ selon les modèles), pour empêcher les grêlons d'atteindre le feuillage et les grappes.

Fabriqués en polypropylène extrudé ou en polyéthylène tissé, éventuellement renforcés par des fils pressés, ils protègent la vigne pendant les tempêtes de grêle du printemps et de l'été, jusqu'à l'époque de la maturation.

Ils sont posés dès le début de la croissance de la vigne, et maintenus par le haut du filet avec des fils en plastique au niveau du haut du palissage, et par d'autres en bas du filet pour assurer une protection face au vent et une certaine rigidité. Ces barres peuvent être relevées lors des travaux en vert, ou de la vendange. Certains dispositifs, dont le coût est plus élevé, sont équipés d'un support enrouleur permettant le remontage du filet avec une manivelle.

Le coût d'installation, en intégrant la main-d'œuvre, est généralement compris selon les modèles entre 10000 et $25000 €$ par hectare (Figs. 5 et 6 ).

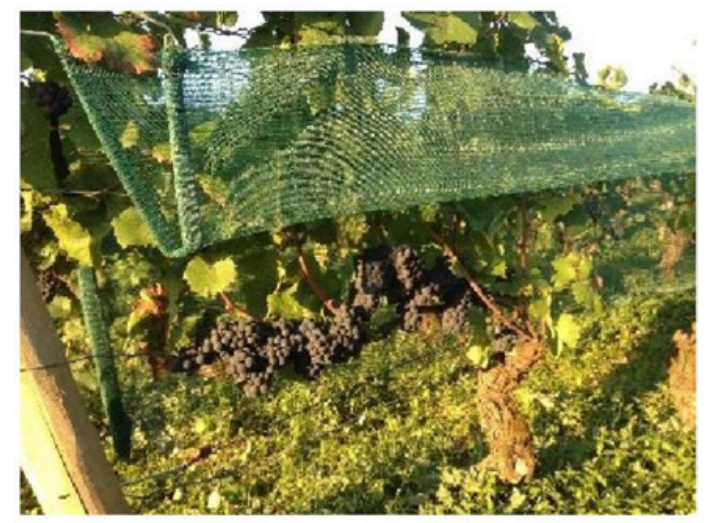

Figure 5. Filet anti-grêle remonté à Morey-St-Denis en Bourgogne (Photo : Christine Monamy).

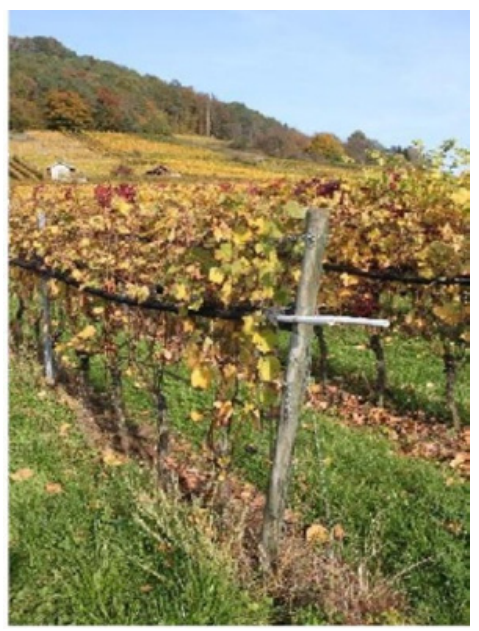

Figure 6. Filet anti-grêle enroulable avec une manivelle (région de Franconie Allemagne).

\section{ASPECT REGLEMENTAIRE DES AOC/AOP}

En France, les dispositifs de protection par filet ont fait l'objet d'une expérimentation menée par la Confédération des Appellations et des Vignerons de Bourgogne pendant plusieurs années. Dans une décision du 20 juin 2018, l'INAO a autorisé l'utilisation des filets de type «monorang vertical» pour la production des vins AOC/AOP, sous réserve que les cahiers des charges des appellations régionales intègrent cette technique, tout en en privilégiant les dispositifs dont la présence peut être limitée dans le temps afin d'atténuer l'effet d'ombrage.

\section{- Protection par ensemencement des nuages}

Le principe de la lutte contre la grêle consiste à introduire artificiellement dans les nuages des noyaux glacogènes d'iodure d'argent de façon à augmenter le nombre de cristaux de glace, et à réduire en conséquence la dimension des grêlons: ceux-ci tombent alors plus lentement et fondent en totalité ou en partie avant d'atteindre le sol (Fig. 7).

L'ensemencement des orages à grêle, mis au point en France par l'ANELFA, est assuré par des réseaux terrestres de générateurs à vortex qui dispersent les noyaux 


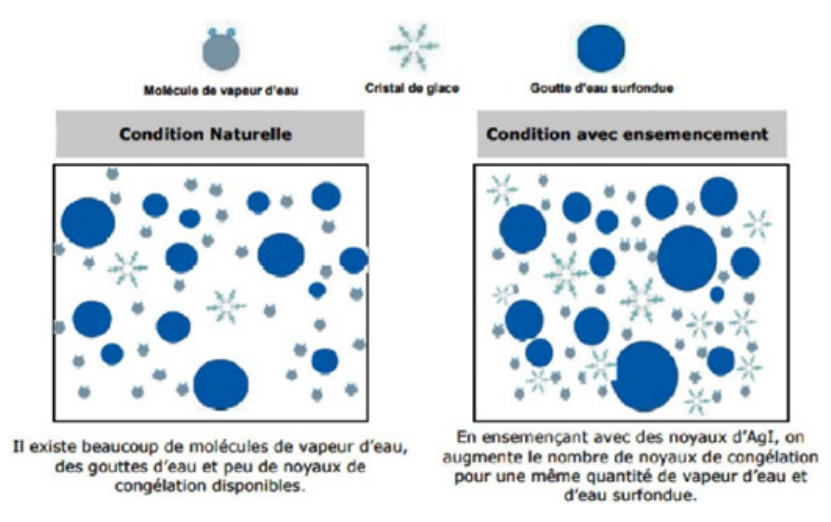

Figure 7. Principes de l'ensemencement anti grêle (Source www. anelfa.asso.fr).

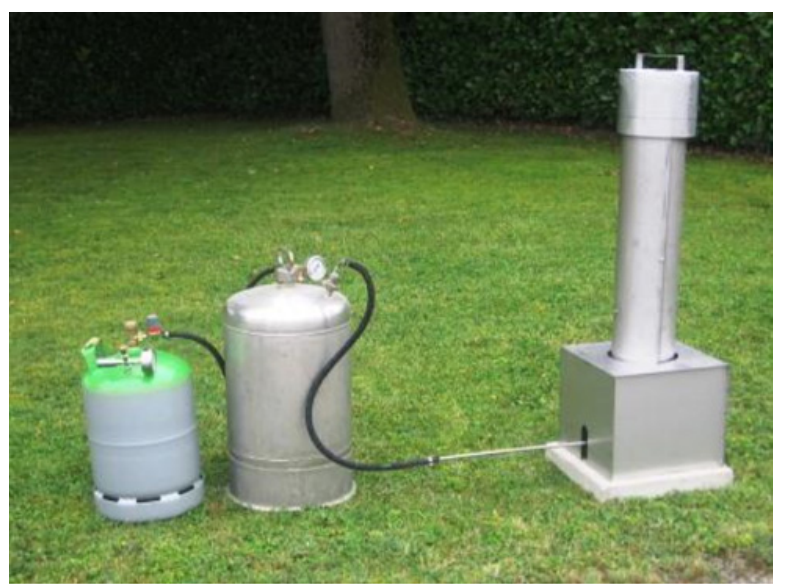

Figure 8. Diffuseur d'Iodure d'Argent (Source ANELFA).

glaçogènes d'iodure d'argent vers les racines des courants ascendants des futurs orages (Fig. 8).

Diverses études permettent de comprendre qu'une action isolée et limitée ne pourra pas avoir d'effet sur un orage et que le dispositif de l'ANELFA sur une large zone, même s'il est plus difficile à mettre en place répond mieux au schéma structurel des orages. La zone à ensemencer dépendant de la vitesse et de la direction de déplacement des orages, les générateurs sont installés sur de larges zones avec un espacement d'environ $10 \mathrm{~km}$ afin de couvrir le maximum de situations.

Un dispositif plus récent «Laïco », encore expérimental, associe un service de détection en temps réel du risque orageux et un nouveau système de diffusion : des ballons gonflés à l'hélium sur lesquels sont embarquées des torches chargées de sels hygroscopiques

\section{Le gel de printemps}

\subsection{Mécanisme et impact}

Les gelées printanières interviennent lorsque la vigne à repris son activité. Les symptômes sont variables et dépendent du stade végétatif. Lors du débourrement, les bourgeons et les rameaux mesurant quelques centimètres brunissent et se dessèchent. Si la gelée est faible, seuls les feuilles hautes et l'apex sont affectés (Fig. 9) [4,5].

La vigne est sensible dès l'apparition des jeunes feuilles qui sont riches en eau. En situation de forte humidité, les jeunes pousses peuvent geler à partir de

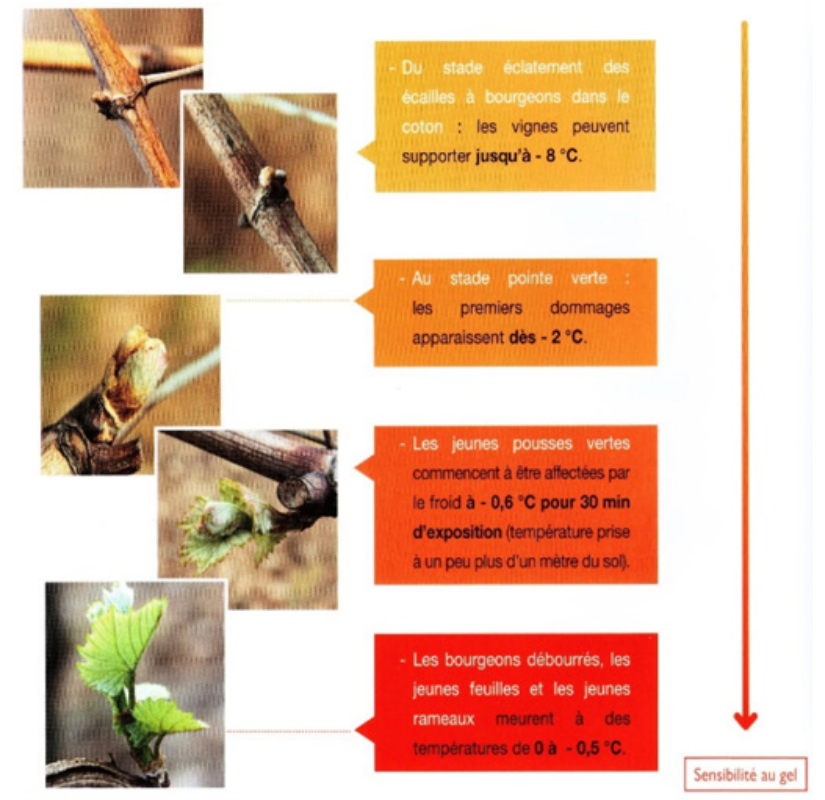

Figure 9. Sensibilité des organes végétaux de la vigne au gel (Source Plaquette les moyens de lutte contre le gel, BIVB, 2018).

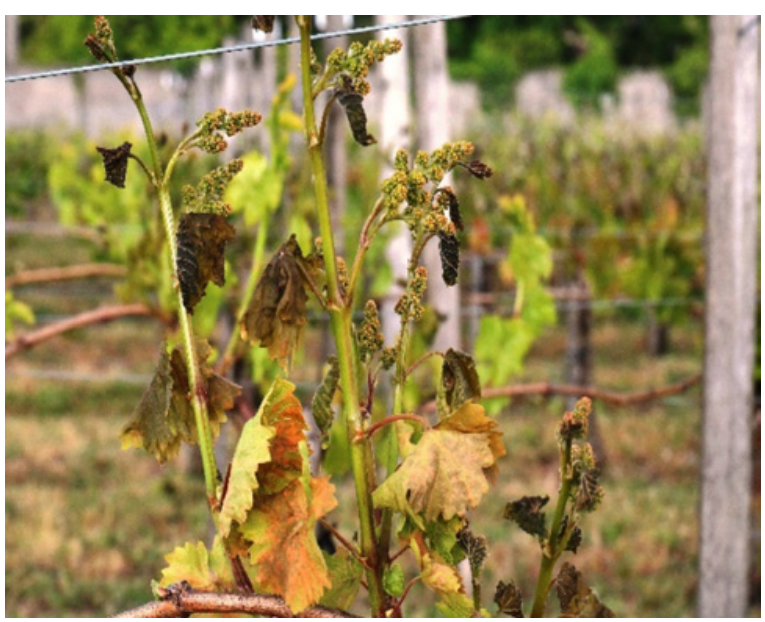

Figure 10. Dégâts de gel à un stade avancé de la vigne (Photo : Jonathan Gaudin/ephytia.inra.fr).

-2 à $-3^{\circ} \mathrm{C}$, alors qu'en situation plus sèche (hygrométrie inférieure à $60 \%$ ), elles peuvent résister à -4 voire $-5^{\circ} \mathrm{C}$. Ces gelées n'entrânent jamais la mort de la vigne. Si la gelée est intense, il peut y avoir destruction totale de la végétation qui brunit après le dégel et prend un aspect de «salade cuite», avant de se dessécher, ce qui peut complexifier la taille de l'année suivante (Fig. 10).

La vigne peut être confrontée à différents contextes de gel. Le refroidissement de l'air peut être associé à l'arrivée d'une masse d'air froid du nord de l'Europe (gel advectif) et plus souvent par temps clair à une perte calorifique du sol non compensée par le rayonnement solaire (gel radiatif) (Fig. 11).

\section{- Gel advectif (gelées noires)}

Rare dans les vignobles français, il est caractérisé par l'arrivée d'une grande masse d'air froid, accompagnée de vent issu généralement du nord-est de l'Europe. Il n'y a pas d'inversion de températures en altitude en raison du vent qui homogénéise les couches d'air. C'est le principal 


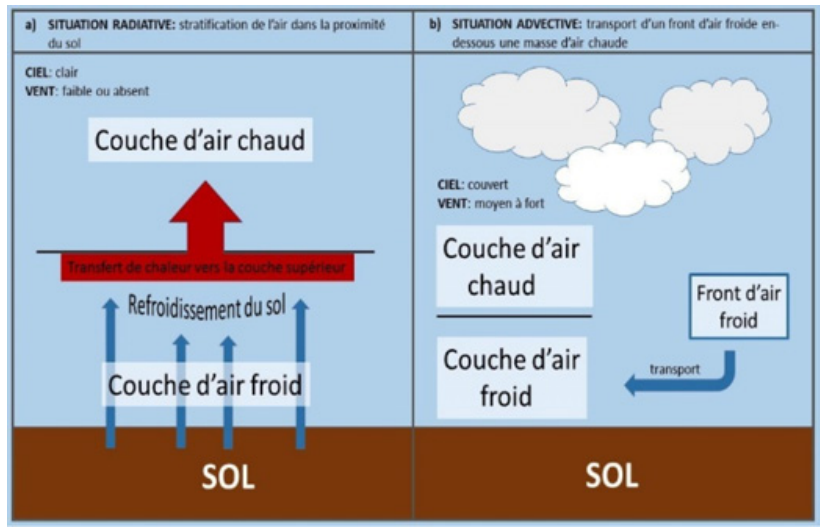

Figure 11. Représentation schématique des processus de formation des gelées radiatif advectif (schéma C. GAVRILESCU, 2016).

mécanisme du gel hivernal, mais il peut également être la cause d'un gel printanier, notamment avec l'avancée de la date de débourrement, en lien avec les changements climatiques.

\section{- Gel radiatif (gelées blanches)}

C'est le type de gel le plus courant, il est caractérisé par une atmosphère stable avec un ciel clair (pas d'effet de serre). Il se produit lors du refroidissement nocturne, avec une présence du vent faible ou nul.

. Si le ciel est nuageux, une partie du rayonnement est captée et retourne vers le sol.

Si le ciel est clair, l'interception des rayonnements par les nuages est impossible et la perte de chaleur est plus grande.

\subsection{Facteurs de gel printanier}

Parallèlement à l'effet de l'altitude (Températures inférieures d'environ 0.6 degré par 100 mètres d'altitude) les gelées sont influencées localement par plusieurs facteurs.

\section{- Topographie}

Les pentes interviennent dans l'écoulement des brises aboutissant à une accumulation d'air froid dans les zones plates ou les cuvettes. Les diverses formes topographiques influencent également la vitesse et la direction du vent. Dans les zones gélives (bas de coteau, fond de vallée) l'air froid, plus lourd que l'air chaud, s'accumule dans une zone à l'abri d'une haie, barrière, forêt ou tout autre obstacle qui empêche l'air froid de s'évacuer (Figs. 12 et 13).

\section{- Humidité}

L'humidité relative, qui croît lorsque la température diminue, augmente la sensibilité des plantes au gel de printemps. Les surfaces humectées préalablement sont plus sensibles aux phénomènes de gel.

\section{- Vent}

Le vent peut fortement limiter la diminution des températures, en brassant les couches atmosphériques (mécanisme utilisé par les brasseurs d'air ou les hélicoptères).

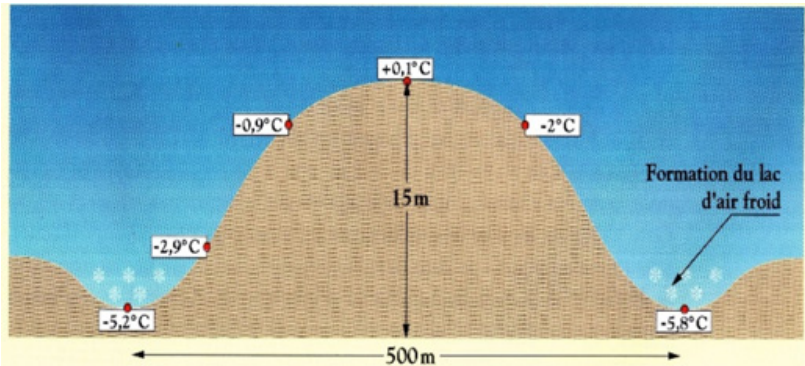

Figure 12. Température minimale observée dans la zone d'Avize en Champagne (Source Le vigneron champenois/CIVC, novembre 1991).

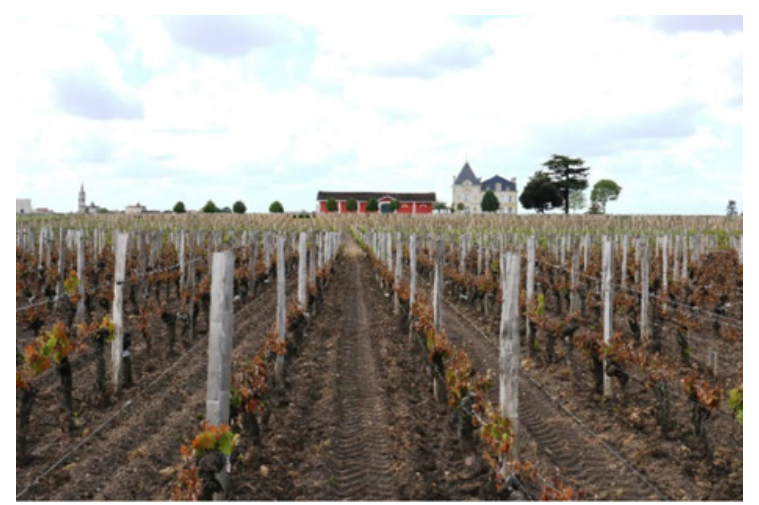

Figure 13. Variabilité de l'impact du gel entre le haut et le bas d'une parcelle du vignoble bordelais. Cette parcelle du bordelais a été partiellement touchée par un épisode de gel. Les ceps affectés présentent des pousses qui brunissent et se dessèchent, tandis que les ceps moins affectés présentent des rameaux restés bien verts. Le secteur touché se situe en contrebas de la parcelle, zone accentuant les effets du gel (Photo : Jonathan Gaudin/ephytia.inra.fr).

\section{- Nébulosité}

La nébulosité favorise l'absorption du rayonnement terrestre par les nuages, et par rediffusion, compense en partie la perte de chaleur par refroidissement radiatif. Par la suite, les températures vont être plus élevées que dans une situation de ciel clair.

\section{- Stade phénologique}

Chaque variété ou clone présente une précocité différente vis-à-vis du débourrement. Le choix d'un cépage avec un débourrement tardif peut limiter les risques dans les zones fortement gélives.

\subsection{Méthodes indirectes de protection}

Les méthodes indirectes que l'on applique bien avant le danger de gel, parfois dès la plantation, sont vraisemblablement les plus économiques et les plus efficaces. Ces mesures concernent notamment:

\section{- Le choix de la parcelle}

Il faut éviter d'installer la vigne dans des zones gélives, particulièrement dans les «creux de terrain» ou les fonds de vallon, dans lesquels l'air froid s'accumule et stagne. La présence de haies compactes, d'arbres ou de bandes 


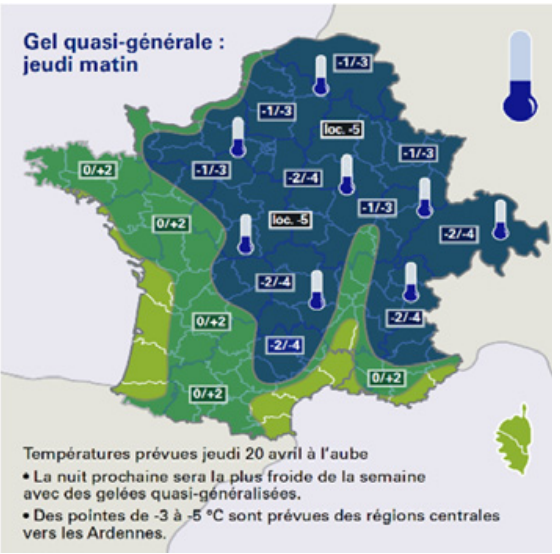

Figure 14. Prévisions météorologiques du 20 avril 2017 (Source meteo-express.com publiée dans le Revue des Enologues, numéro 164, juillet 2017).

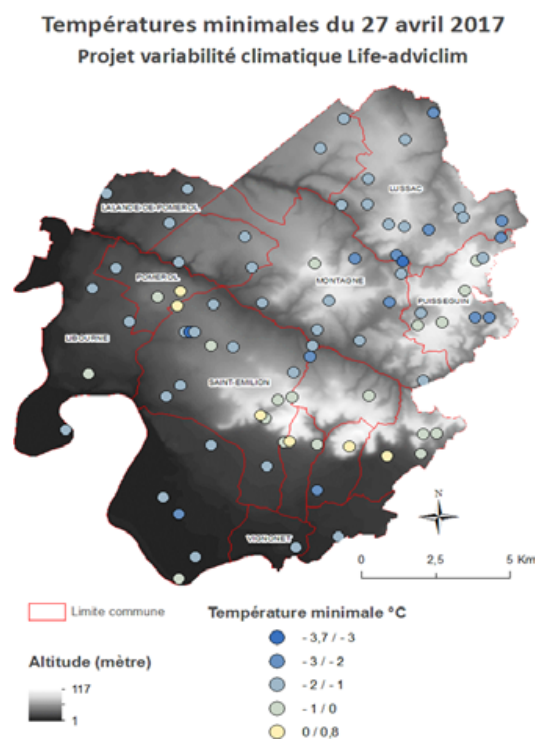

Figure 15. Variabilité de la température au cours de la nuit de gel du 27 avril 2017 dans les régions viticoles de Pomerol, Saint Émilion (Source Laure de Rességuier, Théo Petitjean, Cornelis van Leeuwen www.adviclim.eu).

boisées, en s'opposant à l'écoulement de l'air froid, peut augmenter le risque de gel.

\section{- Les pratiques culturales}

Une tonte avant le débourrement sur les parcelles enherbées peut limiter l'impact du gel. La taille tardive, ainsi que l'attachage de la vigne après la période de risque de gel, sont parfois également utilisés par les vignerons pour limiter le risque.

\subsection{Protection par brassage de l'air Principe}

L'air froid étant le plus dense, on le retrouve au niveau $\mathrm{du}$ sol et de la frondaison de la vigne. La température augmente ensuite sur une épaisseur de quelques mètres pour décroître à nouveau avec l'altitude (en moyenne $6{ }^{\circ} \mathrm{C} / 1000 \mathrm{~m}$ ) au-delà du «plafond d'inversion $\gg$. Le brassage d'air a pour objectif d'assurer une homogénéisation de l'air froid à proximité du sol avec

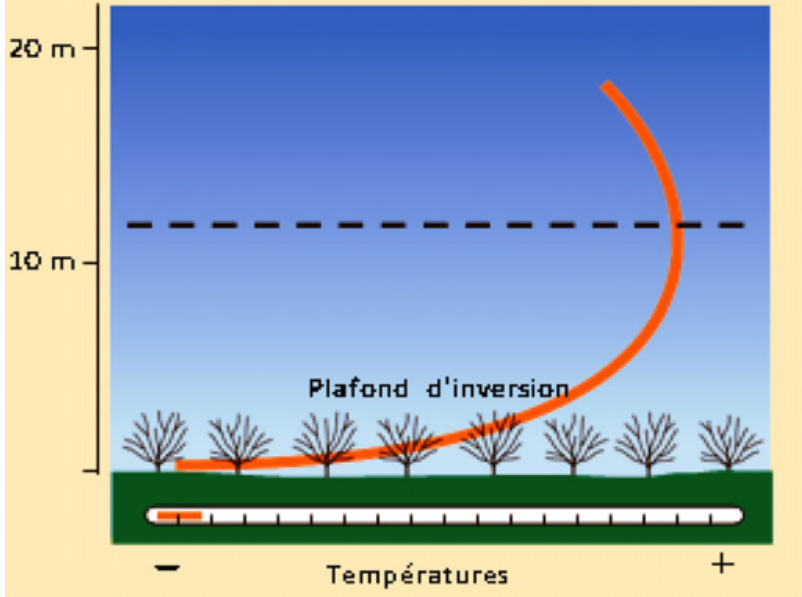

Figure 16. Principe de l'inversion de température en fonction de la hauteur (Source plaquette gel de printemps, chambre d'agriculture du Gard).

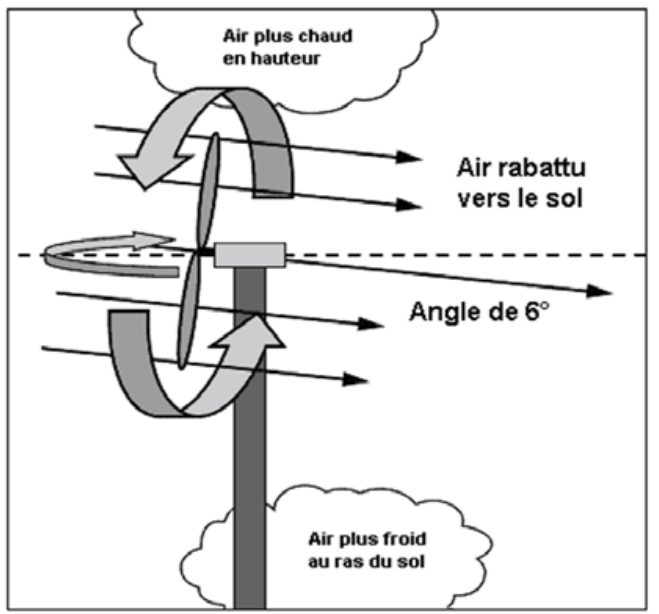

Figure 17. Schéma de principe d'une éolienne antigel (Source www. faiteslepleindavenir.com).

l'air chaud en altitude. Parallèlement à l'aspect thermique, le brassage contribue à un assèchement des bourgeons qui limite leur sensibilité au gel (Fig. 16). Dans le cas de risques de gel de rayonnement, ce brassage peut être provoqué artificiellement par l'utilisation de tours à vent ou d'un hélicoptère (Fig. 17)

\section{- Tours antigel fixes}

Les matériels existant sur le marché sont constitués d'une tour de 10 à 11 mètres de haut surmontée d'une hélice à axe horizontal, légèrement inclinée, avec un angle de 5 à 7 degrés. L'air est aspiré du côté extérieur de l'hélice et refoulé côté intérieur vers le bas. L'axe de l'hélice tourne sur lui-même au rythme d'une rotation de 360" toutes les 4 minutes environ. C'est le temps maximum au-delà duquel la stratification de l'air se remet en place, accompagnée du refroidissement des basses couches d'air (Fig. 18).

Cette technique nécessite peu de main d'œuvre mais génère une nuisance sonore assez importante (70 à $100 \mathrm{~dB}$ à $300 \mathrm{~m})$. Certains brasseurs d'air sont associés 


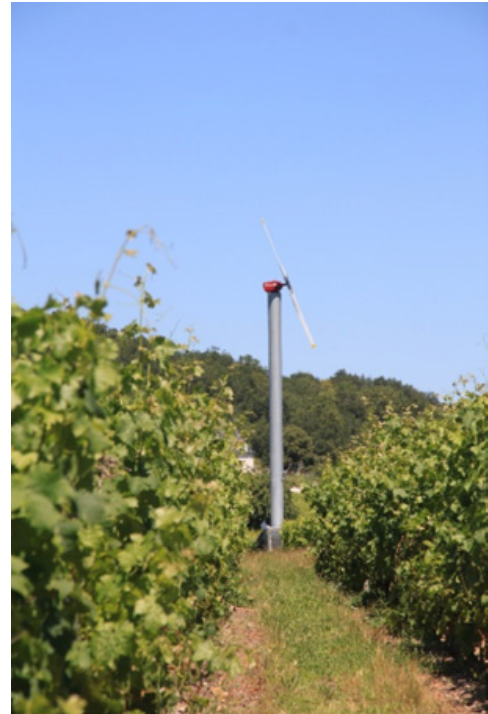

Figure 18. Brasseur d'air classique, région de Chinon en Val de Loire.

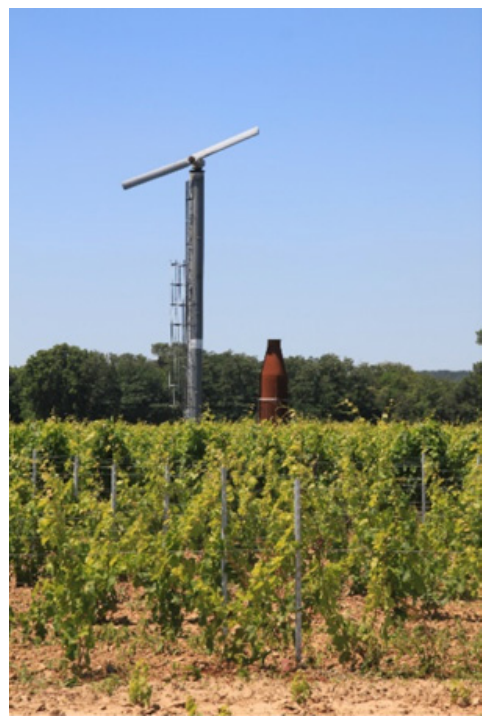

Figure 19. Brasseur d'air avec chauffage, région de Chinon en Val de Loire.

à un dispositif de chauffage qui permet d'optimiser la protection (Fig. 19).

\section{- Tours antigel mobiles}

Ce sont des éoliennes inclinables et éventuellement repliables que l'on peut déplacer selon les besoins. Elles disposent généralement d'un dispositif de démarrage et d'arrêt automatique. Comparativement aux tours fixes, elles sont généralement moins bruyante mais avec une surface de protection inférieure (de l'ordre de 3 ha). Leur mobilité est un atout dans des zones à forte valeur patrimoniale. (Figs. 20 et 21).

\section{- Hélicoptère}

L'hélicoptère, qui doit être mobilisé la veille du gel, rabat au sol la couche d'air chaude situé en altitude et vide les bas-fonds de l'air froid accumulé pendant la

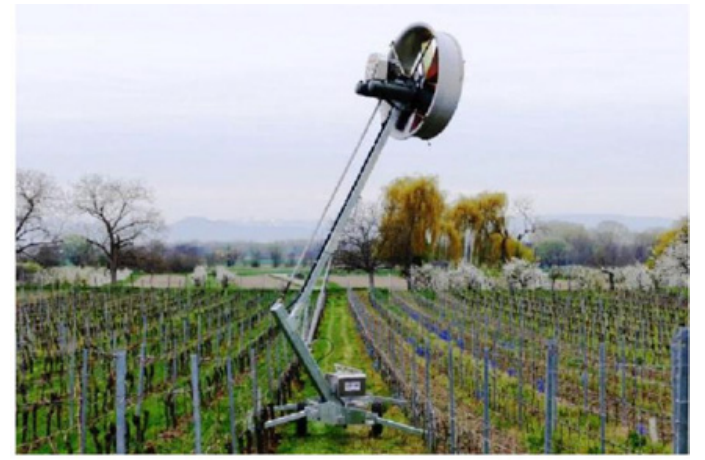

Figure 20. Tour mobile pliable (Photo : www.rivieresarl.com).

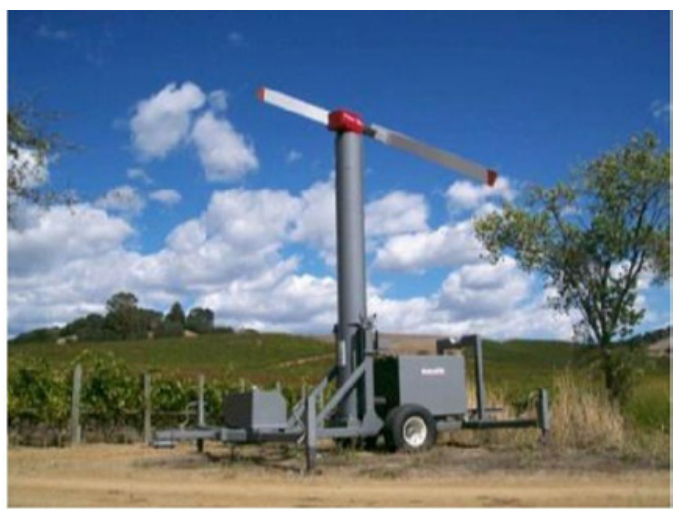

Figure 21. Tour mobile inclinable (Photo : www . filextra.fr).

nuit, (avec un passage au même endroit toutes les 20 minutes). Cette technique peut permettre de protéger entre 20 et 30 hectares en conditions de gel radiatif et en l'absence de vent. La nuisance sonore est importante (de l'ordre de 100 décibels à 300 mètres). Parallèlement, une autorisation de vol est nécessaire pour décoller avant le 《lever du jour aéronautique ». Le coût de la prestation varie généralement de 100 à $300 €$ par hectare pour chaque intervention [7].

\section{- Extraction de l'air froid}

Dans les terrains ondulés, les couches froides s'écoulent par gravité, vers les zones les plus basses de la parcelle. L'air froid s'accumule dans diverses zones en raison de la présence de différents obstacles, tels que diverses barrières végétales, des remblais, ou des variations de pente. Le système «Selective Inverted Sink», jusqu'à présent peu utilisé en Europe, comporte une unité de ventilation horizontale pour extraire l'air stratifié froid du sol, puis l'expulse en dehors de la zone à protéger (Figs. 22 et 23).

\subsection{Dispositifs de chauffage}

\section{- Combustion de gaz}

Ce procédé intervient par réchauffage de l'air et effet de rayonnement dans l'infrarouge. La source d'énergie est le propane qui est distribué aux différents brûleurs sous forme gazeuse à faible pression (de $500 \mathrm{~g} / \mathrm{cm}^{2}$ à $2 \mathrm{~kg} / \mathrm{cm}^{2}$ ). Les brûleurs sont dispensés entre les rangs de vigne à raison dans 150 bruleurs/ha. Ils sont placés au ras du sol et la flamme horizontale est libérée dans une succession 
Tableau 1. Synthèse technico-économique des dispositifs par brassage. Source «Les moyens de lutte contre le gel »BIVB, 2017 [6].

\begin{tabular}{|c|c|c|c|}
\hline & Tour antigel & Hélicoptère & $\begin{array}{l}\text { Aspirateur air } \\
\text { froid } \\
\text { (Sélective } \\
\text { Inverted Sink - } \\
\text { SIS) }\end{array}$ \\
\hline Principe & \multicolumn{2}{|c|}{$\begin{array}{l}\text { Réchauffer l'air froid, plus dense, } \\
\text { situé au niveau des bourgeons en le } \\
\text { mélangeant à de l'air plus chaud } \\
\text { présent en altitude (gel radiatif) }\end{array}$} & $\begin{array}{l}\text { Extraire de l'air } \\
\text { froid plus dense } \\
\text { près du sol. }\end{array}$ \\
\hline \multirow[t]{3}{*}{$\begin{array}{l}\text { Gain } \\
\text { Thermi- } \\
\text { que }\end{array}$} & $\begin{array}{l}\text { Généralement I } \\
{ }^{\circ} \mathrm{C}\left(3^{\circ} \mathrm{C} \text { avec }\right. \\
\text { chauffage } \\
\text { d'appoint })\end{array}$ & $\begin{array}{l}\text { Difficile-ment } \\
\text { évaluable }\end{array}$ & $\begin{array}{l}\text { Jusqu'à } 4{ }^{\circ} \mathrm{C} \\
\text { selon certaines } \\
\text { publications }\end{array}$ \\
\hline & $\begin{array}{l}\text { - peu de main- } \\
\text { d'œuvre et de } \\
\text { surveillance } \\
\text { - protection } \\
\text { jusqu'à des } \\
\text { températures de } \\
-4{ }^{\circ} \mathrm{C} \\
\text { - déclenche- } \\
\text { ment automatisé } \\
\text { - non polluant }\end{array}$ & $\begin{array}{l}\text { - grandes } \\
\text { surfaces } \\
\text { couvertes } \\
\text { (jusqu'à } 20 \text { ha) } \\
\text { - peu de main- } \\
\text { d'œuvre et de } \\
\text { surveillance }\end{array}$ & Pas de référence \\
\hline & $\begin{array}{l}\text { - inefficace } \\
\text { contre le gel } \\
\text { d'advection et } \\
\text { les fortes gelées } \\
\left(-6{ }^{\circ} \mathrm{C} \text { à }-7{ }^{\circ} \mathrm{C}\right) \\
\text { - bruit important } \\
\text { - dépend de la } \\
\text { topographie du } \\
\text { site (site en } \\
\text { cuvette ou faible } \\
\text { pente) } \\
\text { - aspect } \\
\text { paysager }\end{array}$ & $\begin{array}{l}\text { - inefficace } \\
\text { contre les gels } \\
\text { d'advection } \\
\text { - bruit } \\
\text { important } \\
\text { - pas de } \\
\text { décollage avant } \\
7 \text { h } \\
\text { - nécessité } \\
\text { d'autorisa-tions } \\
\text { spéciales } \\
\text { - passage toutes } \\
\text { les } 4 \text { à } 7 \text { mn } \\
\text { pour éviter la } \\
\text { reformation du } \\
\text { gel. }\end{array}$ & $\begin{array}{l}\text { - nécessité } \\
\text { d'avoir un point } \\
\text { bas } \\
\text { d'accumulation } \\
\text { (sinon mise en } \\
\text { place de } \\
\text { barrières } \\
\text { naturelles } \\
\text { possible). } \\
\text { - connexion à un } \\
\text { tracteur ( } 540 \\
\text { tr/min) } \\
\text { - bruit }\end{array}$ \\
\hline Coût & $\begin{array}{l}40000 € \text { pour } 5 \\
\text { ha avec une tour } \\
\text { fixe } \\
47000 € \text { pour } 5 \\
\text { ha avec une tour } \\
\text { pliable } \\
30000 € \text { pour } 4 \\
\text { ha avec une tour } \\
\text { mobile et } \\
\text { pliable } \\
\text { (inefficaces si le } \\
\text { vent }>8 \mathrm{~km} / \mathrm{h} \text { ) }\end{array}$ & $\begin{array}{l}\text { De } 170 \text { à } 220 \\
€ / h a / h\end{array}$ & $\begin{array}{l}13000 \text { à } 26000 \\
€ \text { selon les } \\
\text { modèles. }\end{array}$ \\
\hline
\end{tabular}

de tuyères métalliques, qui servent de plaque rayonnante. Comparativement au fuel le dispositif est moins polluant et présente une plus faible empreinte carbone (Fig. 24).

\section{- Fuel pulvérisé}

Ce procédé comporte en moyenne 180 à 200 brûleurs par hectare, qui consomment au maximum 4001 de fuel par heure, soit la moitié de la quantité nécessaire pour les chaufferettes de grande puissance.

Son installation nécessite généralement une canalisation enterrée en bout de parcelle. Le matériel est placé en

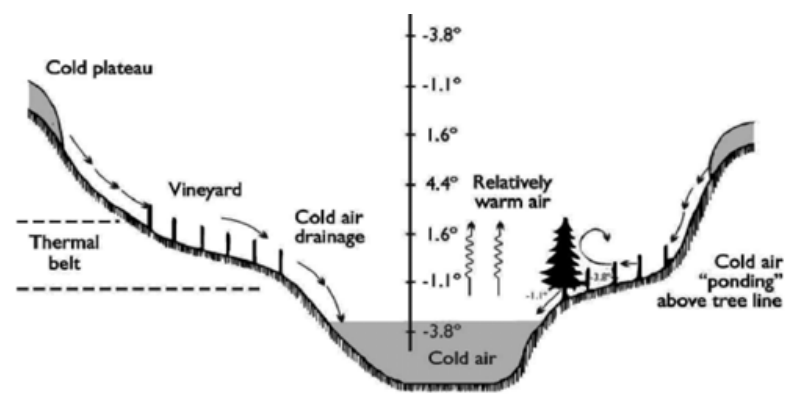

Figure 22. Effet de la topographie du site viticole sur la stratification de la température de l'air pendant une période de refroidissement radiatif caractérisée par des vents calmes et un ciel dégagé. E. Barclay, Poling Hortscience, Vol. 43, october 2008.

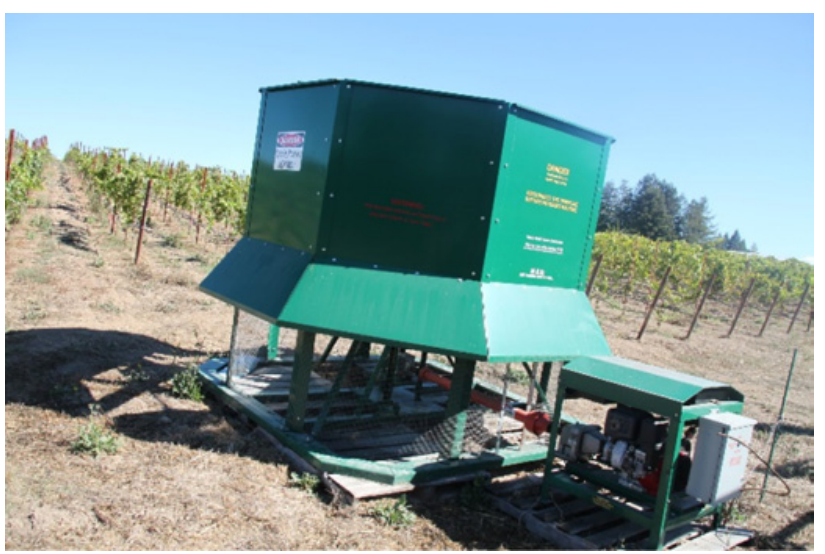

Figure 23. Dispositif « selective inverted sink $»$. Winery DE LOACH dans la Russian River en Californie.

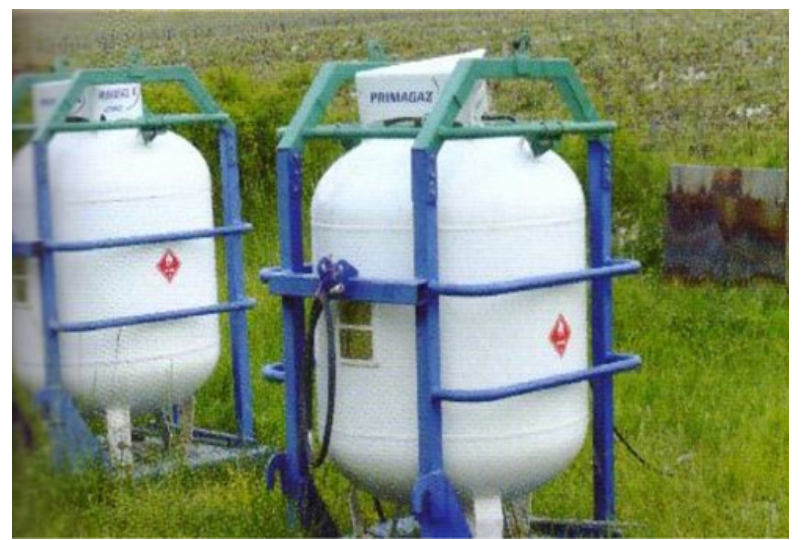

Figure 24. Système de cuves à gaz mobile (Source Guide pratique viticulture durable en Champagne, Le Vigneron champenois 2018 [8]).

début de campagne avant débourrement et déposé après la fin des risques de gelées (fin mai) (Fig. 25).

\section{- Combustible solide}

Ce dispositif se compose de blocs de paraffine pure conditionnée dans une enveloppe métallique ou d'un mélange de paraffine avec différents matériaux combustibles, tels que de la sciure ou des copeaux de bois ou encore de la graisse animale. Généralement 500 unités par hectare permettent un gain thermique compris entre 2 et $2.5^{\circ} \mathrm{C}$. 


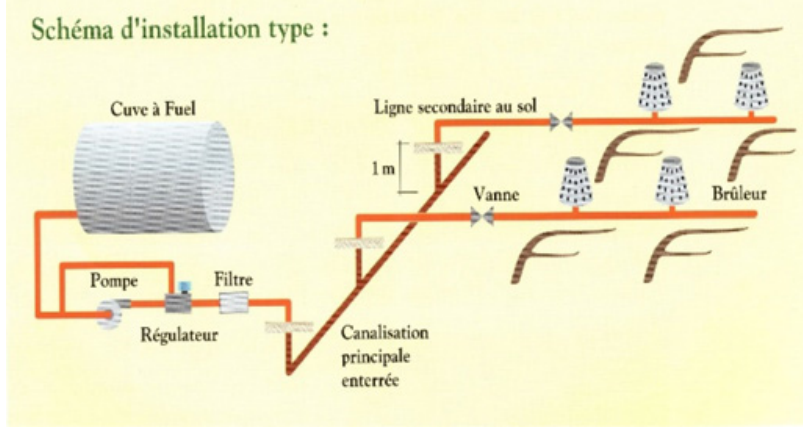

Figure 25. Schéma d'installation d'un dispositif de fuel pulvérisé (Source Les gelées de printemps, le vigneron champenois/CIVC, 1991).

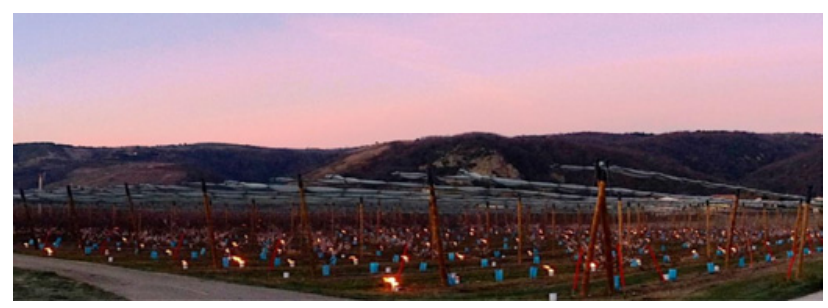

Figure 26. Protection par combustible solide (Photo : Stopgel).

\subsection{Transfert dynamique d'air chaud}

\section{- Frostguard et Frostbuster}

Le dispositif mobile tracté Frostbuster comporte une turbine avec brûleur à gaz qui réchauffe l'air et le diffuse régulièrement sur une distance d'environ 50 mètres de chaque côté. L'air est réchauffé à environ 80 à $100^{\circ} \mathrm{C}$ en sortie de turbine, avec une consommation de l'ordre 40 à $45 \mathrm{~kg}$ de gaz propane à l'heure. Un circuit de circulation dans la parcelle doit être établi pour revenir au même endroit toutes les 7 à 10 minutes au maximum. Il est généralement possible de couvrir une surface de l'ordre de 8 hectares, à condition de pouvoir passer entre les rangs (vignes étroites exclues). Le Frostguard s'inspire du même principe mais reste en poste fixe. Il peut protéger une surface variable selon la topographie ( 0.5 à 0.7 ha $)$ L'investissement est de l'ordre de $8000 €$ par appareil avec une consommation de gaz d'environ $20 €$ par heure de fonctionnement. La nuisance sonore est significative (de l'ordre de 50 décibels à 50 mètres) (Fig. 27).

\section{- Heat Ranger}

Un nouveau dispositif, développé en Nouvelle-Zélande, vient d'être produit en Europe. Il est constitué d'un canon oscillant qui fournit de l'air chaud à partir d'un brûleur au gaz. Le constructeur précise qu'un appareil pourrait protéger une surface de 10 à 20 ha, variable selon la configuration locale et l'intensité du gel (Fig. 28).

\section{- Câbles chauffants}

Les câbles électriques chauffants sont fixés le long du fil de palissage, sur lequel les baguettes et surtout les bourgeons sont alignés. Ce système de lutte peut donc nécessiter

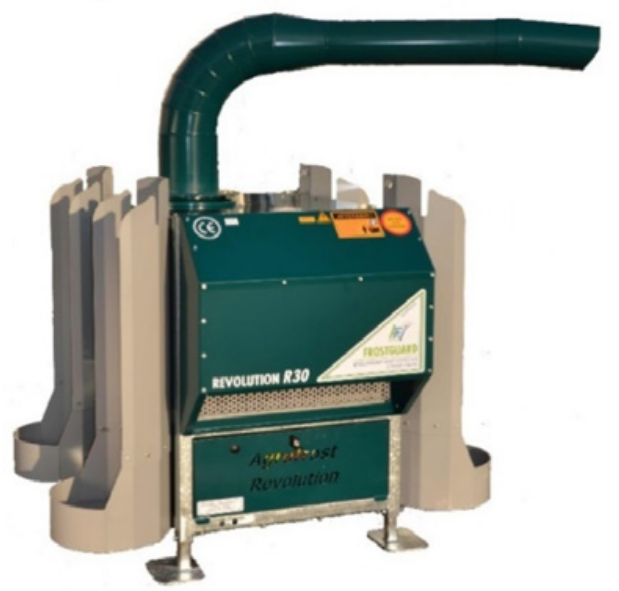

Figure 27. Convecteur d'air chaud fixe FrostGuard (Photo : www. agrofrost.eu).

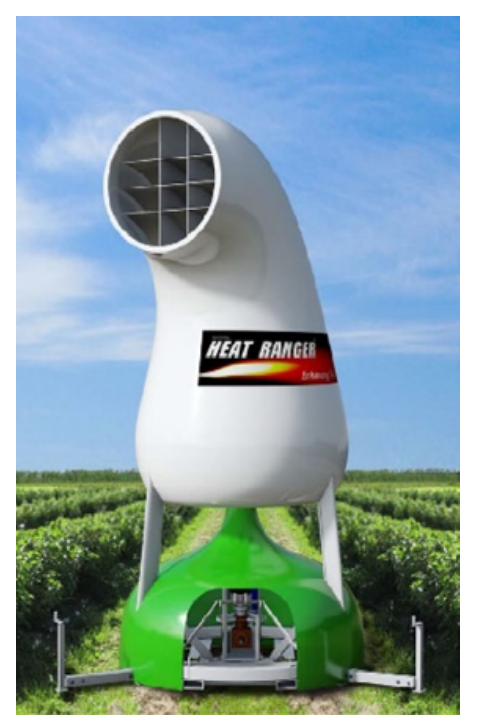

Figure 28. Diffuseur d'air chaud Heat Ranger (Photo : www. heat-ranger. com).

des adaptations de taille (guyot simple ou double, cordon de Royat), car la diffusion du flux de chaleur intervient sur un rayon de 5 à $10 \mathrm{~cm}$. La température de chauffage est comprise entre 28 et $30^{\circ} \mathrm{C}$ et le démarrage peut être automatisé à partir d'un seuil de température.

L'investissement pour des vignes étroites est de l'ordre de 30000 et 40000 euros par hectare (câbles armoires de contrôle, transformateur). Le coût de fonctionnement est assez faible (quelques centaines d'euros par hectare/an), mais il est nécessaire de se relier au réseau électrique et de souscrire un contrat auprès d'un fournisseur, qui doit être négocié au cas par cas. Il est également possible de louer ou d'acheter un/des groupe(s) électrogène(s) puissant(s). Par ailleurs, des précautions doivent être prises pour éviter toute dégradation du câble au cours des travaux de la vigne (taille palissage, vendanges) (Fig. 29).

\section{- Aspersion d'eau}

L'apport d'eau en continu permet d'établir un équilibre eau/glace autour du bourgeon afin de ne pas descendre en dessous de $0{ }^{\circ} \mathrm{C}$. Un dispositif de pompes puissantes 
alimente un réseau d'arroseurs qui quadrille la parcelle. Selon les modèles, les distances entre les rampes et les arroseurs sont généralement comprises entre 15 et 24 mètres. Ce dispositif permet une protection jusqu'à $-7^{\circ} \mathrm{C}$ en conditions humides et $-9^{\circ} \mathrm{C}$ en conditions sèches. La «pluviométrie artificielle» doit être d'au moins $4 \mathrm{~mm} / \mathrm{h}$ avec un débit global d'environ 40 à $60 \mathrm{~m}^{3} / \mathrm{h} / \mathrm{ha} \mathrm{(Fig.} \mathrm{30).}$

Le déclenchement est délicat, car l'évaporation initiale au début de l'arrosage peut contribuer à diminuer la température et accentuer le gel. L'utilisation, dans différentes zones de parcelle, de thermomètre, d'un psychromètre pour mesurer l'humidité de l'air, ainsi qu'un anémomètre peut permettre à une personne expérimentée d'optimiser la protection. D'un point de vue réglementaire et environnemental, cette technique doit prendre en compte notamment le prélèvement d'eau et le risque de ruissellement.

\section{Autres méthodes}

Source : les moyens de lutte contre le gel, BIVB, 2017

\begin{tabular}{|l|l|}
\hline Bâches antigel & $\begin{array}{l}\text { Dans le cas de gel advectif, ce système } \\
\text { peut aggraver la situation par } \\
\text { accumulation d'air froid sous les bâches. }\end{array}$ \\
\hline $\begin{array}{l}\text { Méthode } \\
\text { alternative } \\
\text { substance } \\
\text { élicitrice } \\
\text { (PEL I O I - GV) }\end{array}$ & $\begin{array}{l}\text { - renforcement des défenses naturelles de } \\
\text { la plante (accumulation de diminuant le } \\
\text { point de congélation). Application foliaire } \\
\text { 12 à 48 h avant l'épisode gélif (du stade } \\
\text { bourgeon éclaté à 6 feuilles étalées) } \\
\text { et protection moyenne de 30 à 50\%. } \\
\text { Retours d'expérience épisode de gel en } \\
\text { Bourgogne : efficacité limitée. } \\
\text { - inconvénient : rémanence de 4 jours } \\
\text {-60 €/ha }\end{array}$ \\
\hline $\begin{array}{l}\text { Brûlage paille, } \\
\text { ceps,... }\end{array}$ & $\begin{array}{l}\text { - créer un voile opaque pour limiter le } \\
\text { réchauffement trop rapide au lever du } \\
\text { soleil (effet loupe) et limiter le } \\
\text { rayonnement du sol (si pratiqué la nuit) } \\
\text { avantage : coût réduit } \\
\text { - inconvénients : pollution visuelle et } \\
\text { olfactive, manutention et veille nocturne } \\
\text { nécessaires, non envisageable sur le long } \\
\text { terme. Les particules de fumée étant } \\
\text { trop petites pour bloquer les radiations, } \\
\text { seule la chaleur dégagée par les feux } \\
\text { offre une petite protection. }\end{array}$ \\
\hline $\begin{array}{l}\text { antigel } \\
\text { naturellement présente dans l'atmosphère } \\
\text { et sur les végétaux. Sa membrane contient } \\
\text { une protéine «antigel», qui lui permet de } \\
\text { se protéger contre les cristaux de glace. } \\
\text { Ceux-ci se forment donc à une certaine } \\
\text { distance de la bactérie sans contact direct } \\
\text { avec sa membrane plasmique. Elle serait } \\
\text { donc en partie responsable des gelées sur } \\
\text { les végétaux. L'idée : combattre cette } \\
\text { bactérie par une autre afin de limiter, voire } \\
\text { détruire, sa population. Essais en cours } \\
\text { avec Pseudomonas fluorescens A506 mais } \\
\text { pas d'efficacité prouvée. }\end{array}$ \\
\hline
\end{tabular}

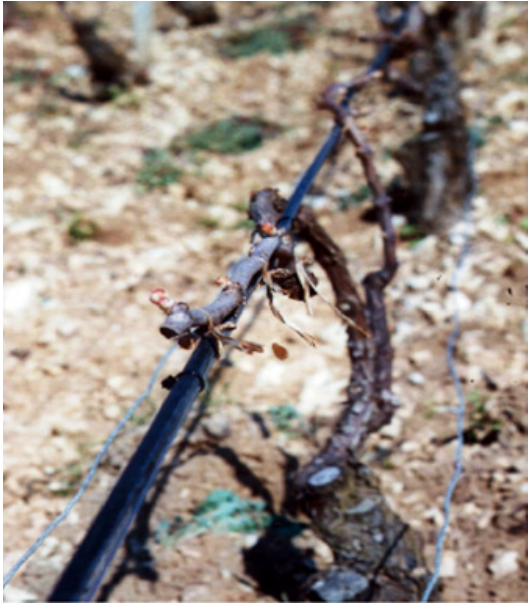

Figure 29. Protection par câble chauffant (Photo : www. technitrace.fr).

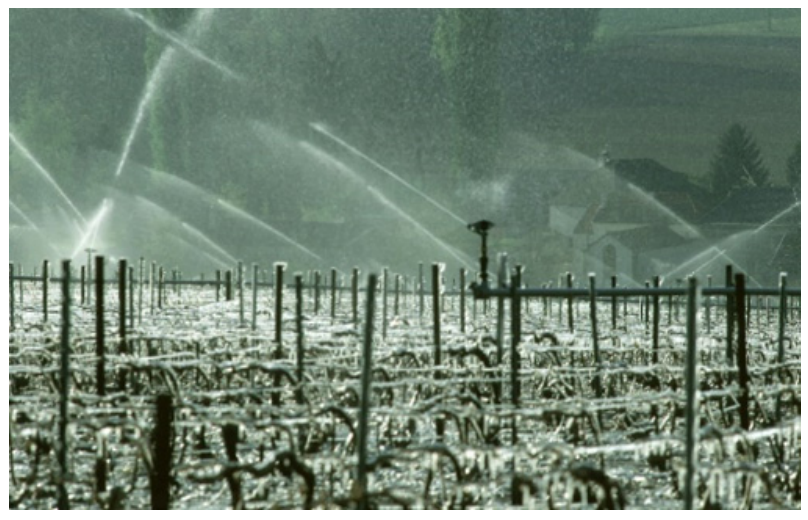

Figure 30. Lutte contre le gel par aspersion en Champagne (Photo : John Hodder/Collection CIVC).

\section{Conclusion}

Les aléas climatiques liés au gel de printemps et grêle peuvent impacter de manière significative l'équilibre financier des exploitations viticoles. Parallèlement aux mesures indirectes (assurance, réserves climatiques) différents systèmes de protection peuvent être envisagés. Les investissements et éventuellement les frais de fonctionnement peuvent être importants, ce qui justifie une réflexion approfondie sur le long terme avant de s'engager. Le choix du dispositif est lié notamment au contexte local (fréquence des aléas, climat, relief), aux contraintes économiques (niveau de valorisation des vins, perte du potentiel commercial) et environnemental (bruit, édition de fumée, risque de ruissellement, impact sur l'effet de serre, etc.).

Concernant l'impact des changements climatiques, contrairement à l'évolution de température, qui peut être mesurée en continu, les phénomènes extrêmes n'interviennent fort heureusement qu'épisodiquement. Il est par conséquent difficile d'établir des statistiques d'évolution sur une période de quelques années. Néanmoins, les climatologues soulignent que l'augmentation de l'évaporation de l'eau en lien avec l'élévation de la température moyenne pourrait conduire à terme à des événements orageux associés à de la grêle, plus fréquents et plus intenses avec une manifestation plus marquée dans les zones septentrionales relativement 


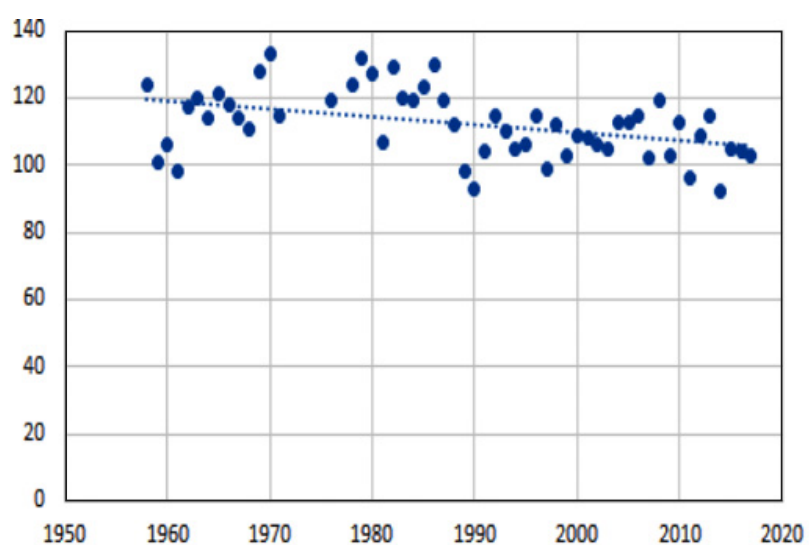

Figure 31. Évolution de la date de débourrement pour le cépage Riesling en Alsace depuis 1958 jusqu'à 2017 (Source E. Duchêne/ INRAColmar).

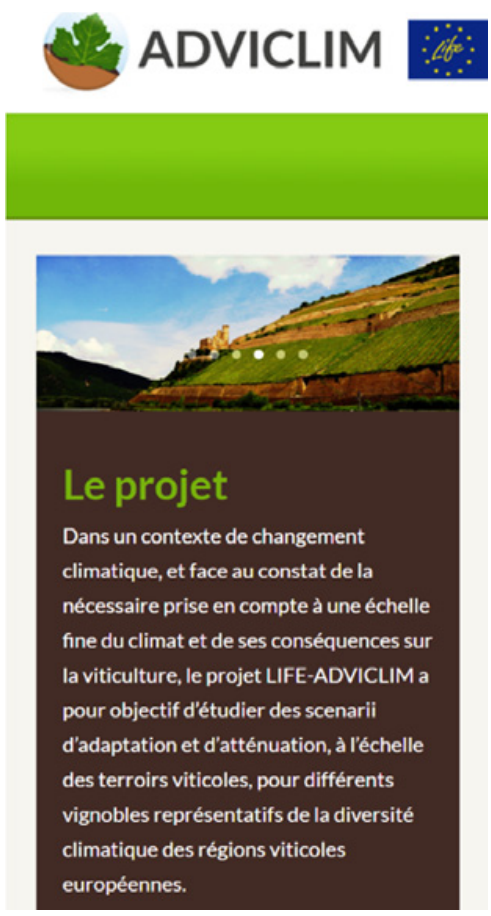

Figure 32. Présentation du projet européen LIFE ADVICLIM www.adviclim.eu.

épargnées jusqu'à présent comparativement aux secteurs méridionaux (Fig. 31).

Vis-à-vis du gel de printemps, l'élévation actuelle de la température moyenne contribue certes à diminuer le nombre de jours avec une température négative durant l'hiver, mais ce réchauffement progressif aboutit également à une avancée du débourrement de la vigne. Ainsi dans les zones septentrionales le gel peut intervenir à partir de la mi-avril et dans les zones méridionales, un gel très destructeur peut se manifester à un stade phénologique avancé.
Cette évolution suppose de développer les connaissances climatiques régionales, et les dispositifs d'avertissement notamment en lien avec un réseau de stations météorologiques, gérés par des organismes institutionnels et/ou professionnels. Parallèlement il peut être intéressant d'avoir une connaissance plus fine à l'échelle des micro terroirs, et éventuellement de la parcelle, notamment pour établir le positionnement et les bases de dimensionnement des dispositifs de protection contre le gel de printemps. C'est une approche qui est développée à l'échelle locale dans le cadre du projet européen ADVICLIM, coordonné par le CNRS.

Nos remerciement à FranceAgrimer qui a financé le projet www.franceagrimer.fr. Que soient également remerciés tous les spécialistes du climat et conseillers viticoles qui ont apporté leur expertise à ce projet et en particulier : Nathalie OLLAT et Jonathan GAUDIN de l'INRA de Bordeaux, Eric DUCHÊNE de l'INRA de Colmar, Benjamin BOIS De l'Université de Bourgogne, INAKI GARCIA de CORTAZAR de l'INRA d'Avignon, Hervé QUENOL du CNRS de Rennes, Jacques GAUTHIER et Philippe DOUMENC de l'INAO, Pierre NAVIAUX du CIVC, Marc OUVRIER de Vineis Project, Vincent DUMOT du BNIC, Philippe ABADIE de la Chambre d'Agriculture de la Gironde, Guillaume MORVAN de la Chambre d'Agriculture de l'Yonne. Un remerciement particulier pour les partenaires du projet européen LIFE ADVICLIM www.adviclim.eu qui ont contribué à une approche internationale de la thématique.

\section{Références}

[1] J. Rochard, Le gel de printemps mi-avril 2017, dans de nombreuses régions européennes Une période très atypique dans les annales de la météo, www.oeno.tm.fr, Revue des œnologues 164, Juillet (2017)

[2] F. VINET, Le risque-grêle en agriculture, éditions technique et documentation (2000)

[3] Grêle: les méthodes de lutte au banc d'essai, Le vigneron des Côtes du Rhône et du SudEst, www . syndicat-cotesdurhone.com, 870, juin (2017)

[4] Gel de printemps en verger, Différents types, seuil critique, moyen de lutte, Chambre d'Agriculture du Gard, https://gard.chambre-agriculture.fr

[5] CTIFL, Gel de printemps, protection des vergers, Ctifl, www.ctifl.fr (1998)

[6] APCA, Protection du vignoble contre les gels de printemps, Chambres d'Agricultures (2018)

[7] BIVB, Les moyens de lutte contre le gel, Bureau Interprofessionnel du Vin de Bourgogne, www . vins-bourgogne.fr (2017)

[8] CIVC, Guide pratique Viticulture durable en Champagne, Le vigneron champenois/CIVC, hors-série (2018) 